\title{
L-carnitine does not improve valproic acid poisoning management: a cohort study with toxicokinetics and concentration/effect relationships
}

Philippe Nguyen ${ }^{1}$, Lucie Chevillard², Ahmed S. Gouda ${ }^{1,3}$, Hervé Gourlain ${ }^{4}$, Laurence Labat ${ }^{4}$, Isabelle Malissin ${ }^{1,2}$, Nicolas Deye ${ }^{1}$, Sebastian Voicu ${ }^{1,2}$ and Bruno Mégarbane ${ }^{1,2^{*}}$

\begin{abstract}
Background: Valproic acid (VPA) poisoning is responsible for life-threatening neurological and metabolic impairments. Despite only low-level evidence of effectiveness, L-carnitine has been used for years to prevent or reverse VPArelated toxicity. We aimed to evaluate the effects of L-carnitine used to treat acute VPA poisoning on the time-course of plasma VPA concentrations and VPA-related toxicity. We designed a single-center cohort study including all VPApoisoned patients admitted to the intensive care unit. We studied VPA toxicokinetics using a nonlinear mixed-effects model-based population approach and modeled individual plasma VPA/blood lactate concentration relationships. Then, we evaluated L-carnitine-attributed effects by comparing VPA elimination half-lives and time-courses of blood lactate levels and organ dysfunction [assessed by the Sequential Organ Failure Assessment (SOFA) score] between matched L-carnitine-treated and non-treated patients using a multivariate analysis including a propensity score.
\end{abstract}

Results: Sixty-nine VPA-poisoned patients (40F/29 M; age, 41 years [32-47]) (median [25th-75th percentiles]; SOFA score, 4 [1-6]) were included. The presumed VPA ingested dose was $15 \mathrm{~g}$ [10-32]. Plasma VPA concentration on admission was $231 \mathrm{mg} / \mathrm{L}$ [147-415]. The most common manifestations were coma (70\%), hyperlactatemia (3.9 mmol/L [2.7-4.9]) and hyperammonemia (127 mmol/L [92-159]). VPA toxicokinetics well fitted a one-compartment linear model with a mean elimination half-life of $22.9 \mathrm{~h}$ (coefficient of variation, 28.1\%). Plasma VPA (C)/blood lactate concentration (E) relationships were well described by an exponential growth equation $\left[E=E_{0} \times e^{k \cdot C}\right.$; with baseline $E_{0}=1.3 \mathrm{mmol} / \mathrm{L}(43.9 \%)$ and rate constant of the effect, $\left.k=0.003 \mathrm{~L} / \mathrm{mg}(59.5 \%)\right]$. Based on a multivariate analysis, peak blood lactate concentration was the only factor independently associated with L-carnitine administration (odds ratio, $1.9,95 \%$ confidence interval, $1.2-2.8 ; P=0.004)$. We found no significant contribution of L-carnitine to enhancing VPA elimination, accelerating blood lactate level normalization and/or preventing organ dysfunction.

Conclusions: VPA poisoning results in severe toxicity. While L-carnitine does not contribute to enhancing VPA clearance, its impact on accelerating blood lactate level normalization and/or preventing organ dysfunction remains uncertain. Investigating VPA toxicokinetics and concentration/effect relationships may help understanding how to improve VPA-poisoned patient management.

\footnotetext{
${ }^{*}$ Correspondence: bruno.megarbane@|rb.aphp.fr

${ }^{1}$ Department of Medical and Toxicological Critical Care, Federation

of Toxicology APHP, Lariboisière Hospital, 2 Rue Ambroise Paré,

75010 Paris, France

Full list of author information is available at the end of the article
}

\section{Springer Open}

(c) The Author(s) 2022. Open Access This article is licensed under a Creative Commons Attribution 4.0 International License, which permits use, sharing, adaptation, distribution and reproduction in any medium or format, as long as you give appropriate credit to the original author(s) and the source, provide a link to the Creative Commons licence, and indicate if changes were made. The images or other third party material in this article are included in the article's Creative Commons licence, unless indicated otherwise in a credit line to the material. If material is not included in the article's Creative Commons licence and your intended use is not permitted by statutory regulation or exceeds the permitted use, you will need to obtain permission directly from the copyright holder. To view a copy of this licence, visit http://creativecommons.org/licenses/by/4.0/. 
Keywords: Valproic acid, Poisoning, L-carnitine, Antidote, Pharmacokinetics, Lactate

\section{Background}

Sodium valproate (VPA), a synthetic 2-propylpentanoic acid, is widely used as an antiepileptic, mood-stabilizer, antipsychotic, anti-migraine and analgesic drug [1]. VPA poisoning may on occasion cause severe toxicity and can very rarely be fatal. Based on the American Association of Poison Control Centers' National Poison Data System, $\sim 7,750$ exposures to VPA including 119 severe and 2 fatal cases were reported in 2019 [2].

VPA poisoning is responsible for central nervous system manifestations ranging from ataxia, sedation and lethargy to coma, respiratory depression, seizures and intracranial hypertension [3]. Various metabolic disorders have been observed including hyperlactatemia and hyperammonemia attributed to VPA-induced alterations in mitochondria functions. Cases with cardiovascular, respiratory, hematological and/or liver failure have been reported and fatalities attributed to brain edema and multi-organ failure [4]. Peak plasma VPA concentration $>450 \mathrm{mg} / \mathrm{L}$ was likely associated with toxicity, while peak VPA $>850 \mathrm{mg} / \mathrm{L}$ with coma, respiratory depression, aspiration and lactic acidosis [5].

In general, VPA-poisoned patients are managed with supportive care alone and gastrointestinal decontamination, if appropriate [6]. Extracorporeal treatment to enhance VPA elimination, preferentially using intermittent hemodialysis, is only recommended in the most severe cases $[7,8]$. Guidelines advise administering L-carnitine in the presence of coma, hyperammonemia and hyperlactatemia attributed to VPA-related toxicity and/or if VPA concentrations are $>850 \mathrm{mg} / \mathrm{L}$ [9]. However, despite its safety and excellent tolerance, evidence to support L-carnitine effectiveness in VPA overdose is scarce relying on anecdotal cases and small case-series $[10,11]$. In the acute poisoning setting, no data support the ability of L-carnitine to alleviate or reverse VPAinduced central nervous system and liver dysfunction [12].

Randomized control trials are needed to evaluate the efficacy and safety of L-carnitine as an antidote, but their feasibility has been questioned due to the rarity of acute VPA poisoning. Therefore, since little is known about L-carnitine-related benefits limiting acute VPA toxicity, we designed this study in VPA-poisoned patients 1 -to describe VPA toxicokinetics; 2 -to analyze the relationships between plasma VPA concentrations and VPA-related effects on lactatemia; and 3-to evaluate L-carnitine effects on VPA elimination, blood lactate level normalization, and organ failure progress.

\section{Methods Study design}

We conducted an 18-year single-center cohort study (2002-2020). The study was conducted according to Helsinki principles, declared to the Commission Nationale de l'Informatique et des Libertés (declaration number, 2067659) and approved by the ethics committee of the French Society of Intensive Care (protocol number, FICS20020231). During ICU stay, appropriate information was given to the patients and next of kin. Written informed consent was waived, since no specific intervention related to the research was performed.

\section{Patient selection and data collection}

All VPA-poisoned adults consecutively admitted to our intensive care unit (ICU) with compatible history and features and at least one plasma VPA concentration $>100 \mathrm{mg} / \mathrm{L}$ (therapeutic range, 40-100) were included. Patients with missing medical records and patients with no available plasma VPA concentration to confirm the diagnosis of VPA poisoning were excluded. Past and recent medical history, comorbidities, presumed VPA ingested doses, co-ingested compounds, clinical presentation, laboratory parameters, toxicological analysis, management, complications and outcome were recorded. The Simplified Acute Physiology Score (SAPS) II [13] was determined on admission. The Sequential Organ Failure Assessment (SOFA) score [14] was calculated on admission and each day during the five following days. Acute kidney injury was graded according to the KDIGO classification [15].

\section{Patient management}

VPA-poisoned patients were managed according to standards of care [9]. Physicians in charge decided plasma sampling to measure plasma VPA concentrations, treatments and L-carnitine dose regimen, which included an intravenous $100 \mathrm{mg} / \mathrm{kg}$ loading dose followed by a maintenance dosing up to $3 \mathrm{~g} /$ day in 3 divided doses for 3 days (or until ICU discharge). L-carnitine was administered based on the recommendations, which did not change during the study period.

\section{Toxicological analyses}

Routine urine and plasma toxicological screening were obtained on ICU admission as performed in all our patients to identify the toxicants to which the patient was exposed. Plasma VPA concentrations were determined using a particle-enhanced turbidimetric immunoassay 
(Alinity c Valproic Reagent Kit ${ }^{\circledR}$, Abbott, Prague, Check Republic; limit of detection, $0.8 \mu \mathrm{g} / \mathrm{mL}$; limit of quantification, $6 \mu \mathrm{g} / \mathrm{mL}$; limit of linearity, $150 \mu \mathrm{g} / \mathrm{mL}$ ). Based on the declared information and toxicological screening results, plasma concentrations of co-ingested toxicants were determined using adequate quantitative assays if available in our institution.

\section{Toxicokinetic modeling}

A population toxicokinetic analysis was performed using a nonlinear mixed-effects model. Only patients with available ingested dose, time from ingestion to admission and at least 2 plasma VPA concentrations were considered. Data were analyzed using NONMEM $^{\circledR}$ version 6.2 (ICON Development Solutions, Ellicott City, MD). The first-order conditional estimation with interaction method was applied. A one-compartment linear model with linear absorption was used to describe VPA concentration-time profiles after oral exposure, as defined by the following equation:

$$
C(t)=\frac{k_{a} \cdot P I D \cdot F}{V d \cdot\left(\frac{C l}{V d}-k_{a}\right)} \cdot\left(e^{-k_{a} \cdot t}-e^{-\frac{C l}{V d} \cdot t}\right)
$$

where $C(\mathrm{t})$ is plasma VPA concentration; $k_{\mathrm{a}}$, the firstorder absorption rate constant; PID, the presumed ingested dose; $F$, the bioavailability after oral exposure; $V_{\mathrm{d}}$ the volume of distribution; and $\mathrm{Cl}$, the total body clearance.

Inter-individual variability was assumed exponential, and the full covariance matrix of the random effects was estimated. Additive, proportional, or mixed error models were tested to describe residual variability. The effects of each patient covariate (i.e., gender, age and L-carnitine treatment) on the toxicokinetic parameters were systematically tested.

The likelihood ratio based on the objective function value was used to test different hypotheses regarding the structure of the variance-covariance matrix for inter-individual variability and residual variability models, and to assess the covariate effects on toxicokinetic parameters.

For evaluation of goodness of fit, we obtained the following three graphs, i.e., population predicted versus observed concentrations, weighted residuals versus time and weighted residuals versus predicted VPA concentrations. Similar graphs using individual predictions were displayed. The population pharmacokinetic parameters are expressed as mean (coefficient of variation).

\section{Concentration-effect relationship modeling}

We investigated VPA-attributed effects on blood lactate level in every individual. Only patients with at least five
VPA/lactate paired values were considered. The relationship of blood lactate level (E) as function of plasma VPA concentration (C) estimated from Eq. 1 was described using the exponential growth equation:

$$
E=E_{0} \times e^{k \cdot C}
$$

where $E_{0}$ is the baseline blood lactate level and $k$, the rate constant of the effect. The model was applied to VPA-attributed effects using the maximum likelihood expectation maximization algorithm implemented in WinNonlin ${ }^{\circledR}$ version 8.02 (Pharsight Corp., Mountain View, CA). All model parameters were assumed lognormally distributed. The model selection was based on goodness-of-fit criteria, which included the convergence criterion, the Akaike information criterion, the estimation criterion value for the maximum likelihood method, and the visual inspection of predicted versus observed and residual plots. Parameters of the concentration-effect relationships are expressed as mean (coefficient of variation) of individual estimations.

\section{Endpoint definitions}

To evaluate L-carnitine effectiveness, we determined the three following endpoints in each patient: (1) VPA elimination half-life $\left(t_{1 / 2}\right)$; (2) the time from admission to normalize blood lactate level; and (3) the deltaSOFA defined as the difference between the worst SOFA score during the first 5 days of ICU stay and the SOFA score on admission.

\section{Statistical analysis}

The qualitative variables are expressed as percentages and the quantitative variables as median [25th75th percentiles]. Comparisons were performed using Mann-Whitney and Fisher exact tests, as appropriate. To analyze L-carnitine-attributed effects, a propensity score to determine the probability for each patient to be treated with L-carnitine was constructed and used in the multivariate analysis. The following confounding factors, i.e., gender, age, blood lactate level on admission, plasma VPA concentration on admission and SOFA score on admission were included in the propensity score calculation. Subsequently, we searched for a relationship between L-carnitine treatment and each endpoint, i.e., the $t_{1 / 2}$, the time for blood lactate level normalization and the deltaSOFA. For each criterion, we performed univariate analyses followed by a multivariate analysis using a linear stepwise regression model, in which significant variables at the $P=0.20$-threshold in the univariate analyses, were included. The propensity score was kept in the final model regardless of its significance levels. The odds ratio and its $95 \%$ confidence interval were calculated for 
every independent parameter associated with L-carnitine treatment. All analyses were carried out with bilateral hypotheses. The statistical analysis was performed using XLStats $^{\circledR} 2017$ software (Addinsoft, New York, NY). $P$-values $<0.05$ were considered as significant.

\section{Results}

\section{Poisoning presentation, management and outcome}

Sixty-nine consecutive VPA-poisoned patients (40F/29 M; age, 41 years [32-47]; body-mass index, $24.2 \mathrm{~kg} / \mathrm{m}^{2}$ [21.3-29.2]) were included. Among these patients, 49 (71\%) were chronically treated with VPA, in relation to epilepsy $(n=29,42 \%)$, bipolar disorder $(n=35,51 \%)$ and psychotic disorder $(n=22,32 \%)$. The presumed VPA ingested dose was $15.0 \mathrm{~g}$ [10.0-32.0], rarely as sustained release formulation $(n=8,14 \%)$. Exposure resulted from multi-drug ingestion $(n=45$, $74 \%)$, involving benzodiazepines $(n=27,39 \%)$, ethanol $(n=10,15 \%)$, antipsychotics $(n=13,19 \%)$ and hypnotics $(n=10,15 \%)$.

On ICU admission, consciousness impairment (Glasgow Coma Score, 6 [3-14]) represented the main clinical manifestation (Table 1). Elevations in blood lactate level $(2.9 \mathrm{mmol} / \mathrm{L}$ [1.8-4.2], normal range, 1.0-2.0) and ammonia (96 $\mu \mathrm{mol} / \mathrm{L}$ [62-132]; normal range, 14-38) represented the main laboratory alterations. Plasma VPA concentration was $231 \mathrm{mg} / \mathrm{L}$ [147-415]. The SOFA score was $4[1-6]$.

During ICU stay, the patients developed coma $(n=48$, $70 \%)$, agitation $(n=12,17 \%)$, seizures $(n=2,3 \%)$ and brain edema evidenced by CT-scan $(n=2,3 \%)$. Blood lactate and serum ammonia concentrations increased in $38 \%$ and $30 \%$ of the patients, peaking at $3.9 \mathrm{mmol} / \mathrm{L}$ [2.74.9] and $127 \mathrm{mmol} / \mathrm{L}$ [92-159], respectively. Plasma VPA concentration increased in $27 \%$ of the patients peaking at $248 \mathrm{mg} / \mathrm{L}$ [147-398] within the first $24 \mathrm{~h}$ from admission in almost all cases. Twenty-three (33\%) patients developed aspiration pneumonia, 13 (19\%) cardiovascular failure and $10(15 \%)$ hospital-acquired infections. Hypoxemia $\left(n=52,75 \% ; \mathrm{PaO}_{2} / \mathrm{FiO}_{2}\right.$ ratio, $297 \mathrm{mmHg}$ [162$389])$, lactic acidosis $(n=17,36 \%)$, mild anemia $(n=15$, $22 \%)$, thrombocytopenia $(n=15,22 \%)$, disseminated intravascular coagulation $(n=2,3 \%)$ and liver cytolysis ( $n=6,9 \%)$ were observed. Acute kidney injury with mild elevation in median serum creatinine $(81 \mu \mathrm{mol} / \mathrm{L}$ [67$102])$ was classified as KDIGO stage $1(n=4,6 \%)$, stage 2 $(n=7,10 \%)$, and stage $3(n=1,2 \%)$.

Overall, management included invasive mechanical ventilation ( $n=41,59 \%$; prior to hospital transfer, $n=34$, $49 \%)$, noninvasive mechanical ventilation $(n=2,3 \%)$, mask oxygen $(n=9,13 \%)$, activated charcoal $(n=32$, $46 \%)$, sedation $(n=32,46 \%)$, intravenous $L$-carnitine $(n=19,28 \%$, administered on ICU admission, once the diagnosis of VPA poisoning was established), norepinephrine $(n=13,19 \%)$, blood transfusion $(n=2,3 \%)$ and hemodialysis $(n=2,3 \%)$. No adverse effect was attributed to L-carnitine administration. The length of ICU stay was 3 days [2-5]. Three patients (4\%) died in the ICU. For additional descriptive data of particular subgroups, see Additional file 1: Table S1.

\section{Population toxicokinetics}

VPA toxicokinetics well fitted a one-compartment linear model with linear absorption $(n=19)$. The model parameters are presented in Table 2. The mean VPA $t_{1 / 2}$ was $22.9 \mathrm{~h}(28.1 \%)$ and the mean apparent clearance 1.2 $\mathrm{L} / \mathrm{h}$ (22.1\%). The predicted versus observed VPA concentrations, the weighted residuals versus time and the weighted residuals versus predicted VPA concentrations are presented in Fig. 1. The individual model-predicted versus observed VPA concentrations are presented in Fig. 2.

\section{Plasma VPA/blood lactate concentration relationships}

The relationships between blood lactate and plasma VPA concentrations well fitted an exponential growth equation $(n=8)$. The model parameters are shown in Table 2 . The model-predicted versus observed values are shown in Fig. 3. The time-course of VPA-induced effects on blood lactate concentrations are shown in Fig. 4.

\section{Analysis of L-carnitine benefits in VPA poisoning}

Based on univariate analyses, L-carnitine-treated and non-treated patients significantly differed regarding coma onset ( $95 \%$ versus $60 \%, P=0.007$ ), blood bicarbonate $(22 \mathrm{mmol} / \mathrm{L}$ [19-24] versus $23 \mathrm{mmol} / \mathrm{L}$ [20-26], $P=0.003)$, blood lactate level $(3.3 \mathrm{mmol} / \mathrm{L}$ [2.0-4.7] versus $2.7 \mathrm{mmol} / \mathrm{L}$ [1.7-4.1], $P=0.0013)$, hemoglobin (14 g/ $\mathrm{dL}$ [12-16] versus $13 \mathrm{~g} / \mathrm{dL}$ [12-14], $P=0.043)$, white blood cell count $(6.1 \mathrm{G} / \mathrm{L}$ [4.8-8.5] versus $7.1 \mathrm{G} / \mathrm{L}$ [5.49.4], $P=0.027$ ) (see more details in Table 1).

The construction of the propensity score is shown in Additional file 1: Table S2. In contrast to age, gender, SOFA score and VPA concentration on admission, the only parameter kept in the propensity score associated with L-carnitine administration was blood lactate level on admission (odds ratio, 1.31 [0.98-1.75], $P=0.068$ ). Based on the multivariate analysis, the peak blood lactate level was the only parameter associated with L-carnitine administration (odds ratio, 1.9 [1.2-2.8]; $P=0.004$; Additional file 1: Table S3).

In the univariate analyses, the delay to normalize blood lactate (21 h [13-24] versus $7 \mathrm{~h}[0-18], P=0.03$ ) differed between L-carnitine-treated and non-treated patients, whereas $t_{1 / 2}(20 \mathrm{~h}$ [16-27] versus $18 \mathrm{~h}$ [12$25], P=0.1)$ and the deltaSOFA (1.0 versus $1.0, P=0.6$ ) 
Table 1 Clinical and laboratory parameters in 69 valproic acid-poisoned patients on admission to the intensive care unit and univariate comparisons according to L-carnitine administration

\begin{tabular}{|c|c|c|c|c|}
\hline & $\begin{array}{l}\text { All patients } \\
(\mathrm{n}=69)\end{array}$ & $\begin{array}{l}\text { Patients managed } \\
\text { without L-carnitine }(n=50)\end{array}$ & $\begin{array}{l}\text { Patients managed } \\
\text { with L-carnitine } \\
(n=19)\end{array}$ & $p$-value \\
\hline \multicolumn{5}{|l|}{ Demographics and medical history } \\
\hline Age (years) & $41[32-47]$ & $40[34-47]$ & 40 [29-48] & 0.70 \\
\hline Weight (kg) & $69[62-81]$ & $68[60-80]$ & $68.0[63-91]$ & 0.96 \\
\hline Size $(\mathrm{cm})$ & 168 [162-173] & 165 [160-170] & $170[160-174]$ & 0.54 \\
\hline Body-mass index $\left(\mathrm{kg} / \mathrm{m}^{2}\right)$ & $24.2[21.3-29.2]$ & $24[31-22]$ & 23 [19-26] & 0.94 \\
\hline Gender (F/M), $n(\%)$ & $40(58 \%) / 29(42 \%)$ & $29(58 \%) / 21(42 \%)$ & $11(58 \%) / 8(42 \%)$ & 1.00 \\
\hline Mood disorder, $n(\%)$ & $35(51 \%)$ & $29(58 \%)$ & $6(32 \%)$ & 0.063 \\
\hline Epilepsy, n (\%) & $29(42 \%)$ & $21(42 \%)$ & $8(42 \%)$ & 1.00 \\
\hline Psychotic disorder, $n$ (\%) & $22(32 \%)$ & $14(28 \%)$ & $8(42 \%)$ & 0.39 \\
\hline Metabolic pathology, n (\%) & $8(12 \%)$ & $7(14 \%)$ & $1(5 \%)$ & 0.43 \\
\hline Heart disease, $n(\%)$ & $6(9 \%)$ & $5(10 \%)$ & $1(5 \%)$ & 1.00 \\
\hline Liver disease, $n$ (\%) & $4(6 \%)$ & $4(8 \%)$ & $0(0 \%)$ & 0.57 \\
\hline Diabetes mellitus, $n(\%)$ & $2(3 \%)$ & $0(0 \%)$ & $2(10 \%)$ & 0.07 \\
\hline Chronic renal failure, $n(\%)$ & $2(3 \%)$ & $2(4 \%)$ & $0(0 \%)$ & 1.00 \\
\hline Long-term VPA treatment, $n(\%)$ & $49(71 \%)$ & $34(68 \%)$ & $15(79 \%)$ & 0.55 \\
\hline Sustained release VPA, $n(\%)$ & $8(14 \%)$ & $3(7 \%)$ & $5(33 \%)$ & 0.026 \\
\hline Co-intoxications, $n(\%)$ & $45(74 \%)$ & $33(75 \%)$ & $12(71 \%)$ & 0.75 \\
\hline Presumed dose ingested (g) & $15.0[10.0-32.0]$ & $15.6[10.0-31.0]$ & $10.0[3.0-30.0]$ & 0.91 \\
\hline \multicolumn{5}{|l|}{ Clinical parameters on ICU admission } \\
\hline Glasgow coma score & $6[3-14]$ & $6[3-13]$ & $8[3-14]$ & 0.24 \\
\hline Temperature $\left({ }^{\circ} \mathrm{C}\right)$ & $36.8[32.2-37.3]$ & $36.8[36.2-37.5]$ & $36.7[36.1-37.2]$ & 0.18 \\
\hline Systolic blood pressure $(\mathrm{mmHg})$ & 119 [110-133] & 119 [110-133] & 113 [101-130] & 0.67 \\
\hline Diastolic blood pressure $(\mathrm{mmHg})$ & $64[57-74]$ & $64[56-73]$ & $66[50-75]$ & 0.035 \\
\hline Heart rate (/min) & $94[82-104]$ & 93 [82-102] & $95[74-104]$ & 0.11 \\
\hline Respiratory rate (/min) & 19 [16-22] & 19 [16-21] & $20[16-24]$ & 0.48 \\
\hline \multicolumn{5}{|l|}{ Laboratory parameters on ICU admission } \\
\hline Serum creatinine $(\mu \mathrm{mol} / \mathrm{L})$ & $73[65-86]$ & $74[62-87]$ & $70[61-80]$ & 0.39 \\
\hline Arterial pH & $7.39[7.36-7.43]$ & $7.39[7.36-7.43]$ & $7.37[7.36-7.44]$ & 0.55 \\
\hline $\mathrm{HCO}_{3}^{-}(\mathrm{mmol} / \mathrm{L})$ & $23[20-26]$ & $23[20-26]$ & $22[19-24]$ & 0.003 \\
\hline $\mathrm{PaO}_{2} / \mathrm{FiO}_{2}(\mathrm{mmHg})$ & 355 [262-467] & 355 [281-470] & $308[105-450]$ & 0.63 \\
\hline AST (IU/L) & $27[20-42]$ & $25[21-45]$ & $24[20-36]$ & 0.43 \\
\hline ALT (IU/L) & $19[11-31]$ & $19[11-30]$ & $17[10-31]$ & 0.84 \\
\hline Prothrombin index (\%) & 86 [77-94] & 86 [78-94] & 83 [72-91] & 0.71 \\
\hline Bilirubin (UI/L) & $7[5-11]$ & $6[5-11]$ & $7[5-10]$ & 0.17 \\
\hline White blood cells (G/L) & $7.1[5.4-9.3]$ & $7.1[5.4-9.4]$ & $6.1[4.8-8.5]$ & 0.027 \\
\hline Platelets (G/L) & $202[168-251]$ & $196[163-251]$ & 199 [184-234] & 0.36 \\
\hline Hemoglobin (g/dL) & $13.3[12.2-14.4]$ & $13[12-14]$ & $14[12-16]$ & 0.043 \\
\hline Blood lactate level (mmol/L) & $2.9[1.8-4.2]$ & $2.7[1.7-4.1]$ & $3.3[2.0-4.7]$ & 0.001 \\
\hline Blood ammonia level (mmol/L) & $96[62-132]$ & 83 [38-129] & $96[40-131]$ & 0.30 \\
\hline Plasma VPA concentration (mg/L) & $231[147-415]$ & $210[143-358]$ & 274 [174-607] & 0.11 \\
\hline \multicolumn{5}{|l|}{ Physiological scores on ICU admission } \\
\hline SOFA score & $4[1-6]$ & $4[2-6]$ & $5[1-5]$ & 0.59 \\
\hline SAPS II & $32[20-41]$ & $30[18-41]$ & $35[23-40]$ & 0.35 \\
\hline \multicolumn{5}{|l|}{ Peak laboratory parameters } \\
\hline Peak blood lactate level (mmol/L) & $3.7[2.5-4.9]$ & $3.5[2.5-4.9]$ & $4.6[3.0-6.1]$ & 0.002 \\
\hline Peak blood ammonia level (mmol/L) & 127 [92-248] & $124[48-275]$ & $227[147-302]$ & 0.19 \\
\hline Peak plasma VPA concentration (mg/L) & 249 [150-398] & $231[147-365]$ & 287 [174-779] & 0.06 \\
\hline
\end{tabular}


Table 1 (continued)

\begin{tabular}{|c|c|c|c|c|}
\hline & $\begin{array}{l}\text { All patients } \\
(n=69)\end{array}$ & $\begin{array}{l}\text { Patients managed } \\
\text { without L-carnitine }(n=50)\end{array}$ & $\begin{array}{l}\text { Patients managed } \\
\text { with L-carnitine } \\
(\mathrm{n}=19)\end{array}$ & $p$-value \\
\hline \multicolumn{5}{|l|}{ Complications in the ICU } \\
\hline Coma, $n(\%)$ & $48(70 \%)$ & $30(60 \%)$ & $18(95 \%)$ & 0.007 \\
\hline Seizures, $n(\%)$ & $2(3 \%)$ & $2(4 \%)$ & $0(0 \%)$ & 1.00 \\
\hline Agitation, n (\%) & $12(17 \%)$ & $9(18 \%)$ & $3(18 \%)$ & 1.00 \\
\hline Brain edema, $n(\%)$ & $2(3 \%)$ & $0(0 \%)$ & $2(11 \%)$ & 0.073 \\
\hline Hypotension, n (\%) & $20(29 \%)$ & $14(28 \%)$ & $6(32 \%)$ & 0.77 \\
\hline Tachycardia, n (\%) & $20(29 \%)$ & $13(26 \%)$ & $7(37 \%)$ & 0.39 \\
\hline Cardiovascular failure, $n(\%)$ & $14(20 \%)$ & $8(14 \%)$ & $6(32 \%)$ & 0.41 \\
\hline Lactic acidosis, $n$ (\%) & $17(36 \%)$ & $12(38 \%)$ & $5(33 \%)$ & 0.31 \\
\hline Liver cytolysis, n (\%) & $6(9 \%)$ & $4(8 \%)$ & $2(11 \%)$ & 0.66 \\
\hline Thrombocytopenia, $n$ (\%) & $15(22 \%)$ & $9(18 \%)$ & $6(32 \%)$ & 0.33 \\
\hline Anemia, $n(\%)$ & $15(22 \%)$ & $8(16 \%)$ & $7(37 \%)$ & 0.099 \\
\hline Disseminated intravascular coagulation & $2(3 \%)$ & $1(2 \%)$ & $1(5 \%)$ & 0.48 \\
\hline Acute kidney injury, $n(\%)$ & & & & 0.10 \\
\hline Stage 1 & $4(6 \%)$ & $2(4 \%)$ & $2(11 \%)$ & \\
\hline Stage 2 & $7(10 \%)$ & $4(8 \%)$ & $3(16 \%)$ & \\
\hline Stage 3 & $1(2 \%)$ & $0(0 \%)$ & $1(5 \%)$ & \\
\hline Aspiration pneumonia, $n(\%)$ & $23(33 \%)$ & $14(28 \%)$ & $9(47 \%)$ & 0.16 \\
\hline Hospital-acquired infection, $n(\%)$ & $10(15 \%)$ & $6(12 \%)$ & $4(21 \%)$ & 0.45 \\
\hline \multicolumn{5}{|l|}{ Treatments in the ICU } \\
\hline Activated charcoal & $32(46 \%)$ & $20(40 \%)$ & $12(63 \%)$ & 0.11 \\
\hline $\begin{array}{l}\text { Ventilation } \\
\text { Non-invasive ventilation } \\
\text { Invasive ventilation }\end{array}$ & $\begin{array}{l}11(16 \%) \\
41(59 \%)\end{array}$ & $\begin{array}{l}10(20 \%) \\
25(50 \%)\end{array}$ & $\begin{array}{l}1(5 \%) \\
16(84 \%)\end{array}$ & 0.091 \\
\hline Norepinephrine, $n$ (\%) & $13(19 \%)$ & $7(14 \%)$ & $6(32 \%)$ & 0.16 \\
\hline Sedation, $n(\%)$ & $32(46 \%)$ & $22(44 \%)$ & $10(53 \%)$ & 0.59 \\
\hline Hemodialysis, $n(\%)$ & $2(3 \%)$ & $0(0 \%)$ & $2(11 \%)$ & 0.073 \\
\hline Transfusion, $n(\%)$ & $2(3 \%)$ & $1(2 \%)$ & $1(5 \%)$ & 0.48 \\
\hline $\begin{array}{l}\text { Outcome, } n(\%) \\
\text { Home } \\
\text { Medical ward } \\
\text { Psychiatry department } \\
\text { Death }\end{array}$ & $\begin{array}{l}29(43 \%) \\
7(10 \%) \\
29(43 \%) \\
3(4 \%)\end{array}$ & $\begin{array}{l}22(45 \%) \\
5(10 \%) \\
21(43 \%) \\
1(2 \%)\end{array}$ & $\begin{array}{l}7(37 \%) \\
2(11 \%) \\
8(42 \%) \\
2(11 \%)\end{array}$ & 0.49 \\
\hline Length of ICU stay (days) & $3[2-5]$ & $2[2-5]$ & $5[2-6]$ & 0.06 \\
\hline
\end{tabular}

Significant $p$-values $(<0.05)$ are noted in bold

ALT alanine aminotransferase, AST Aspartate aminotransferase, ICU intensive care unit, SAPS I/ Simplified Acute Physiology Score II, SOFA Sequential Organ Failure Assessment, VPA valproic acid. Acute kidney injury was staged based on KDIGO classification

did not. In the multivariate analysis, no significant effect of L-carnitine administration was found on $t_{1 / 2}$, the delay to normalize blood lactate level and the deltaSOFA.

\section{Discussion}

VPA poisoning is responsible for severe and even fatal presentations including coma, hyperlactatemia and hyperamonemia. L-carnitine administration was not associated with significant alteration in VPA elimination nor significant clinical or metabolic benefit.

\section{Mechanisms of VPA toxicity}

Due to similar structures, VPA and medium-chain fatty acids are at risk of metabolic competition [4, 16, 17]. Multiple metabolic pathways are involved in VPA biotransformation, giving rise to $\geq 50$ known metabolites. VPA undergoes mitochondrial $\beta$-oxidation and to a lesser extent microsomal $\omega$-oxidation. Additional involved metabolic pathways include hydroxylation, 
Table 2 Parameters of the population toxicokinetic model of plasma valproic acid and parameters of the model of plasma valproic acid/blood lactate concentration relationships in valproic acid-poisoned patients managed in the intensive care unit

\begin{tabular}{llc}
\hline Parameters & Mean & CV\% \\
\hline Population toxicokinetic model $(n=19)$ & & \\
$\mathrm{k}_{\mathrm{a}}(/ \mathrm{h})$ & 0.5 & Fixed \\
$\mathrm{V}_{\mathrm{d}} / \mathrm{F}(\mathrm{L})$ & 39.9 & 28.1 \\
$\mathrm{Cl} / \mathrm{F}(\mathrm{L} / \mathrm{h})$ & 1.2 & 22.1 \\
$\mathrm{ke}(/ \mathrm{h})$ & 0.03 & 28.1 \\
$\mathrm{t}_{1 / 2}(\mathrm{~h})$ & 22.9 & 28.1 \\
$\eta_{(\mathrm{V} / \mathrm{F})}(\%)$ & 1.1 & 30.7 \\
$\eta_{(\mathrm{Cl} / \mathrm{F})}(\%)$ & 0.9 & 32.3 \\
$\varepsilon_{\text {prop }}$ & 0.4 & 23.0 \\
Relationships between blood lactate and plasma valproic acid concentra- \\
tions $(\mathrm{n}=8)$ & & \\
$\mathrm{E}_{0}(\mathrm{mmol} / \mathrm{L})$ & 1.3 & 43.9 \\
$\mathrm{~K}(\mathrm{~L} / \mathrm{mg})$ & 0.003 & 59.5 \\
\hline
\end{tabular}

$\mathrm{k}_{\mathrm{a}}$ absorption rate constant; $\mathrm{V}_{\mathrm{d}} / \mathrm{F}$, apparent volume of distribution; $\mathrm{Cl} / \mathrm{F}$, apparent total body clearance; $k_{\mathrm{e}}$ elimination rate constant; $\mathrm{t}_{1 / 2}$, elimination half-life; $\eta_{(V / F)}$, inter-individual variability in the apparent volume of distribution; $\eta_{(C / / F)}$, inter-individual variability in the apparent clearance; $\varepsilon_{\text {prop }}$ proportional error of the model; $\mathrm{E}_{0}$, baseline blood lactate level; $\mathrm{k}$, rate constant of the effect; $\mathrm{CV} \%$, coefficient of variation

glucuronidation and other minor conjugation reactions. VPA acyl-CoA esters formed in the cytosol by oxidation enter the mitochondria via the carnitine shuttle.

VPA-related toxicity results from the accumulation of some toxic metabolites, which may result from the misbalance between the intra-mitochondrial $\beta$-oxidation and the microsomal $\omega$-oxidation, in relation to VPA transport blockage across the mitochondrial membrane [17]. These toxic metabolites contribute to the loss of mitochondrial membrane potential, modulate the activity of selected enzymes and transport systems, impair mitochondrial fatty acid $\beta$-oxidation and inhibit urea cycle [17, 18]. All these mitochondrial dysfunctions lead to VPArelated metabolic disorders including hyperammonemia, hyperlactatemia and liver microvesicular steatosis. Our findings clearly support relationships between lactate elevation and plasma VPA concentrations.

\section{Clinical consequences of VPA toxicity}

In our series, the main VPA poisoning manifestation consisted of consciousness impairment, observed in $70 \%$ of the patients. However, severity of consciousness impairment in relation to the observed plasma VPA concentrations should be interpreted with cautious considering the multidrug ingestions mainly involving psychotropic drugs and the development of tolerance in the acute-on-chronically poisoned patients. Interestingly, we did not found significant differences when comparing acutely and acute-on-chronically VPA-poisoned patients, although we could not rule out an underpowered analysis. Two patients developed brain edema, which represents the most serious VPA-attributed neurological complication $[3,5]$. Of note, in one of these two patients, edema occurred even though initial VPA concentration was moderately high $(190 \mathrm{mg} / \mathrm{L})$ but rapidly increased (up to $832 \mathrm{mg} / \mathrm{L}$ ). This observation supports the necessity of repeating plasma VPA measurements despite initial reassuring values, due to prolonged absorption in overdose, especially with slow-release formulations. Both patients with brain edema were treated with L-carnitine and presented favorable outcome without neurological sequelae.

Interestingly, our findings also support a large interindividual variability regarding VPA-attributed effects on blood lactate concentrations as suggested by the elevated coefficients of variation of our pharmacodynamic model parameters (Table 2). However, the exact reasons for such a variability, which may correspond to patients with enhanced vulnerability to VPA toxicity, remains to be investigated.

\section{VPA toxicokinetics}

Pharmacokinetics of VPA have been studied extensively, characterized by a high inter-individual variability [1]. In summary, gastrointestinal absorption is almost complete ( $\geq 80 \%$ bioavailability) allowing plasma concentration peaking before 2 or $8 \mathrm{~h}$ post-ingestion with the immediate versus sustained release formulation, respectively. Plasma protein binding is extensive (90-95\%), increasing with age and decreasing at higher VPA concentrations. The volume of distribution ranges from 8.4 to $23.3 \mathrm{~L}$ using one-compartment models and 4.08 to $42.1 \mathrm{~L}$ using two-compartment models. VPA elimination follows firstorder kinetics with a 0.206 to $1.154 \mathrm{~L} / \mathrm{h}$ clearance. An average $t_{1 / 2}$ of 10-12 $\mathrm{h}$ has been determined, with a range of 4-17 h. VPA undergoes extensive biotransformation, mainly in the liver, including mitochondrial $\beta$-oxidation (accounting for $\geq 40 \%$ of the dose and involving three cytochrome P450 enzyme isoforms CYP2C9, CYP2A6 and CYP2B6), other oxidative mechanisms $(<15-20 \%)$ and glucuronide conjugation (30-50\%). Less than $5 \%$ of the ingested amount is eliminated unchanged in urine.

By contrast, very few toxicokinetic investigations exist. A population pharmacokinetic approach with Bayesian estimation, as performed in our VPA-poisoned patients, allows incorporating several factors affecting VPA kinetics into individualized drug therapy. In acute poisonings, absorption was shown to be rapid with peak concentrations observed 3.5-5.6 h 


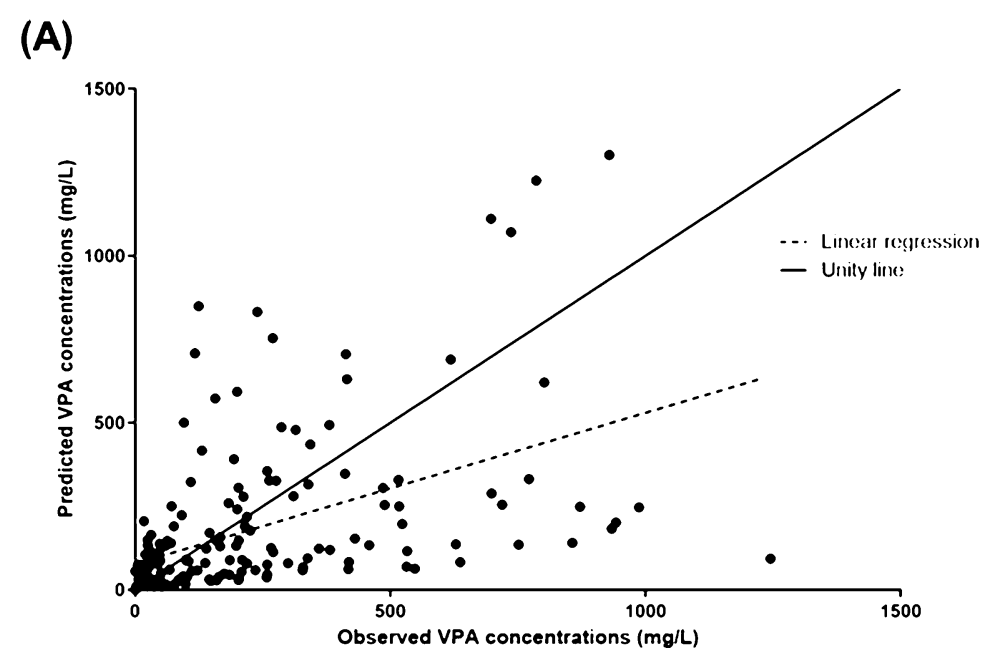

(B)

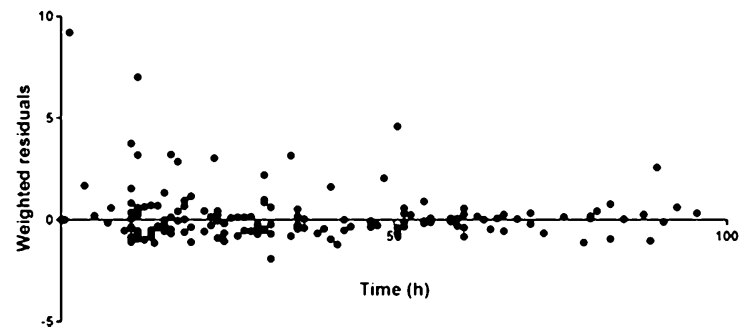

(C)

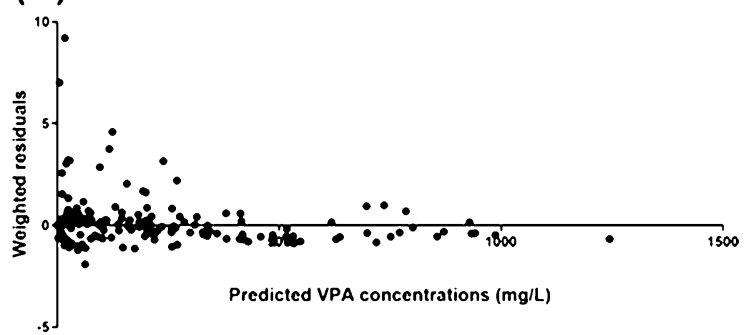

Fig. 1 Validation of the valproic acid (VPA) toxicokinetic model in nineteen poisoned patients with the predicted versus observed plasma VPA concentrations (A), the weighted residuals versus time $(\mathbf{B})$ and the weighted residuals versus predicted plasma VPA concentrations $(\mathbf{C})$

post-ingestion [19]. VPA time-course was found biphasic, with $t_{1 / 2}$ of $8.8-30.9 \mathrm{~h}$ and an apparent volume of distribution of $0.17-0.72 \mathrm{~L} / \mathrm{kg}$. The time-course of plasma VPA was studied later in another cohort of 20 VPA-poisoned patients using a two-compartment population pharmacokinetic model [20]. VPA therapy was shown to increase the $\mathrm{V}_{\max }$ of $\beta$-oxidation by $59 \%$. The observed differences in VPA elimination between the overdose and therapeutic settings were attributed to $\beta$-oxidation pathway saturation due to its MichaelisMenten kinetics.

In our series, most of our patients were in the elimination phase on admission fitting a one-compartment linear model. This observation may in part be related to the fact that immediate-release formulations were predominantly ingested but may also support the benefits of activated charcoal administration including at repeated doses as carried out in almost half of our patients. Our $t_{1 / 2}$, clearance and volume of distribution were consistent with values found in overdose elsewhere $[19,20]$. Our mildly prolonged $t_{1 / 2}$ despite preserved clearance might correspond to the distribution volume increase in comparison to pharmacological conditions or to $\beta$-oxidation pathway saturation, as suggested [20]. Interestingly, gastrointestinal decontamination was shown to moderately lower the bioavailability by an average of $34 \%$ [20].

\section{Effectiveness of L-carnitine in VPA poisoning}

Carnitine is an essential cofactor in VPA metabolism allowing its transport across the mitochondria membrane and permitting ammonia elimination. Hypocarnitinemia results from urine excretion of valproylcarnitine, decreased tubular reabsorption of carnitine and inhibition of endogenous carnitine production, thus increasing blood acyl-carnitine/free carnitine ratio $[16,18]$. A lack of carnitine is thought to contribute to hyperammonemia and L-carnitine, the levorotatory form of carnitine, was suggested as possible treatment to lower ammonemia in long-term VPA-treated patients [21]. Experimental and clinical data suggest that early L-carnitine supplementation can improve the outcome of VPA-induced hepatotoxicity in chronically treated patients $[18,22]$. L-carnitine supplementation 
effectively reversed VPA-induced hyperammonemic encephalopathy [23], despite the absence of correlation between its severity, plasma VPA and serum ammonia concentrations [16, 24]. Moreover, prophylactic supplementation had also been advocated in VPA-treated epileptic children at high-risk of toxicity [12]. By extension, because of its role in restoring beta-oxidation metabolism in the liver cell mitochondria, L-carnitine was proposed in severely VPA-poisoned patients, who present lactic acidosis, liver failure, brain edema, and/ or VPA concentrations of $>850 \mathrm{mg} / \mathrm{L}$.

In our department, the decision to administer L-carnitine was left to the physician in charge. The proportion of patients receiving L-carnitine did not change over time during the study period. Interestingly, we found that peak blood lactate was the only factor independently associated with L-carnitine infusion. This observation supported the fact that $\mathrm{L}$-carnitine prescription in our ICU was consistent with the national guidelines [9]. We observed no side effects attributed to L-carnitine, consistent with previous reports establishing the excellent tolerance of L-carnitine, such as the study showing no adverse effects in relation to $251 \mathrm{~L}$-carnitine doses administered in the setting of VPA toxicity [25].

Curiously, evidence to support effectiveness of L-carnitine in improving VPA poisoning is still poor. Reduction in $t_{1 / 2}$ was attributed to L-carnitine in eight non-overdosed long-term VPA-treated patients $(9.5 \mathrm{~h}$ versus $12 \mathrm{~h}$, $P<0.05)$ [26]; however, $t_{1 / 2}$ in this study were shorter compared to ours. A Romanian randomized controlled trial including 62 VPA-poisoned patients admitted to the ICU (28 treated versus 34 non-treated patients with $\mathrm{L}$-carnitine $1,800 \mathrm{mg} /$ day for 3 days) reported that L-carnitine reduced plasma VPA levels and facilitated the decrease in plasma ammonia concentrations [27]. However, this study was only published as a congress abstract
A
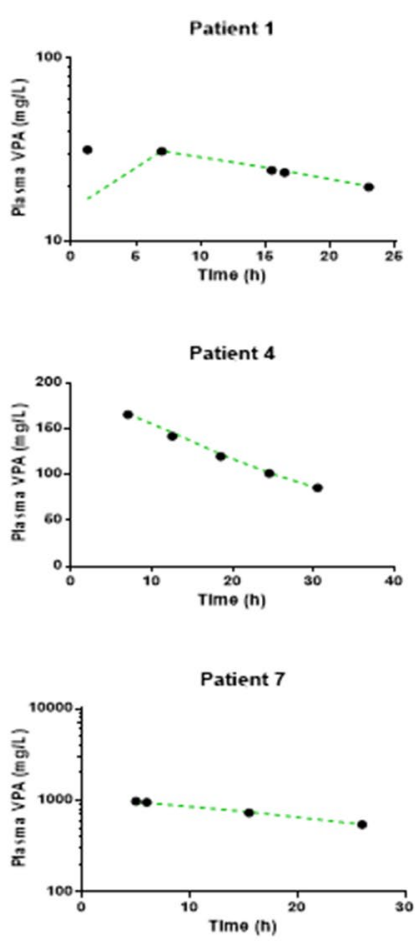

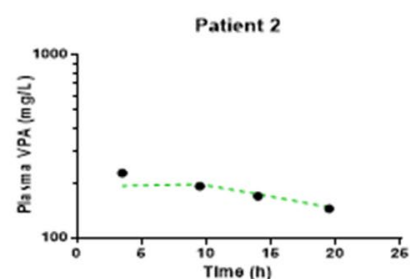

Patient 5

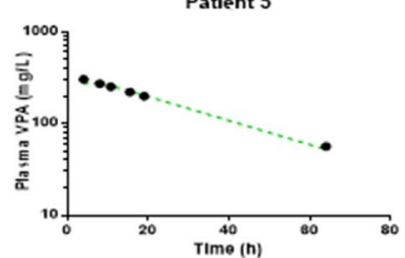

Patient 8

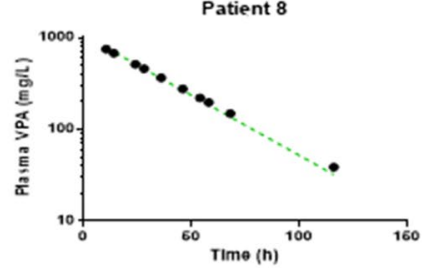

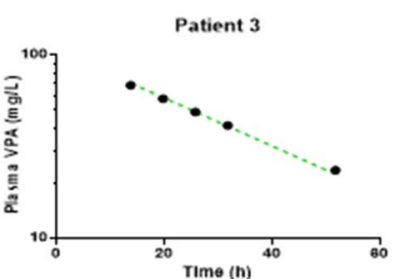

Patient 6
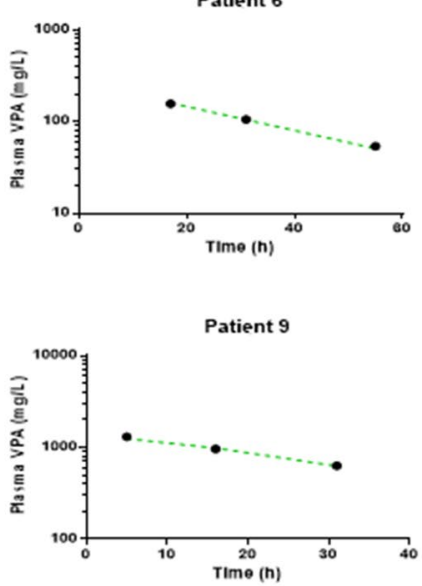
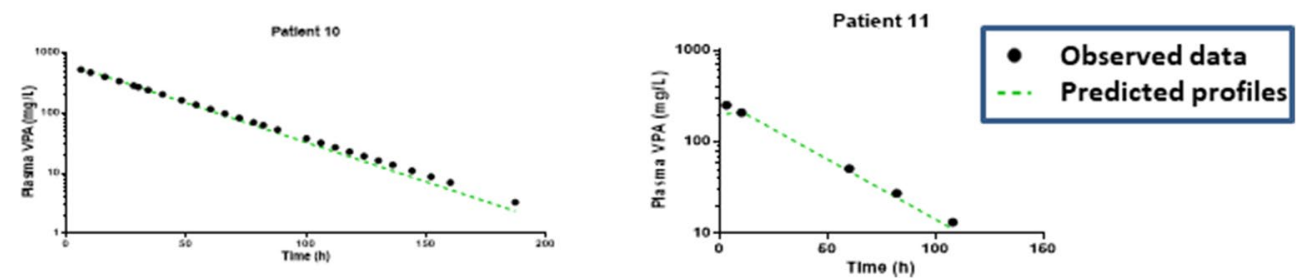

Fig. 2 Individual toxicokinetics showing the best fit of the observed values in nineteen valproic acid (VPA)-poisoned patients non-treated (panel A) or treated with L-carnitine (designated as LC, panel B) 


\section{B}
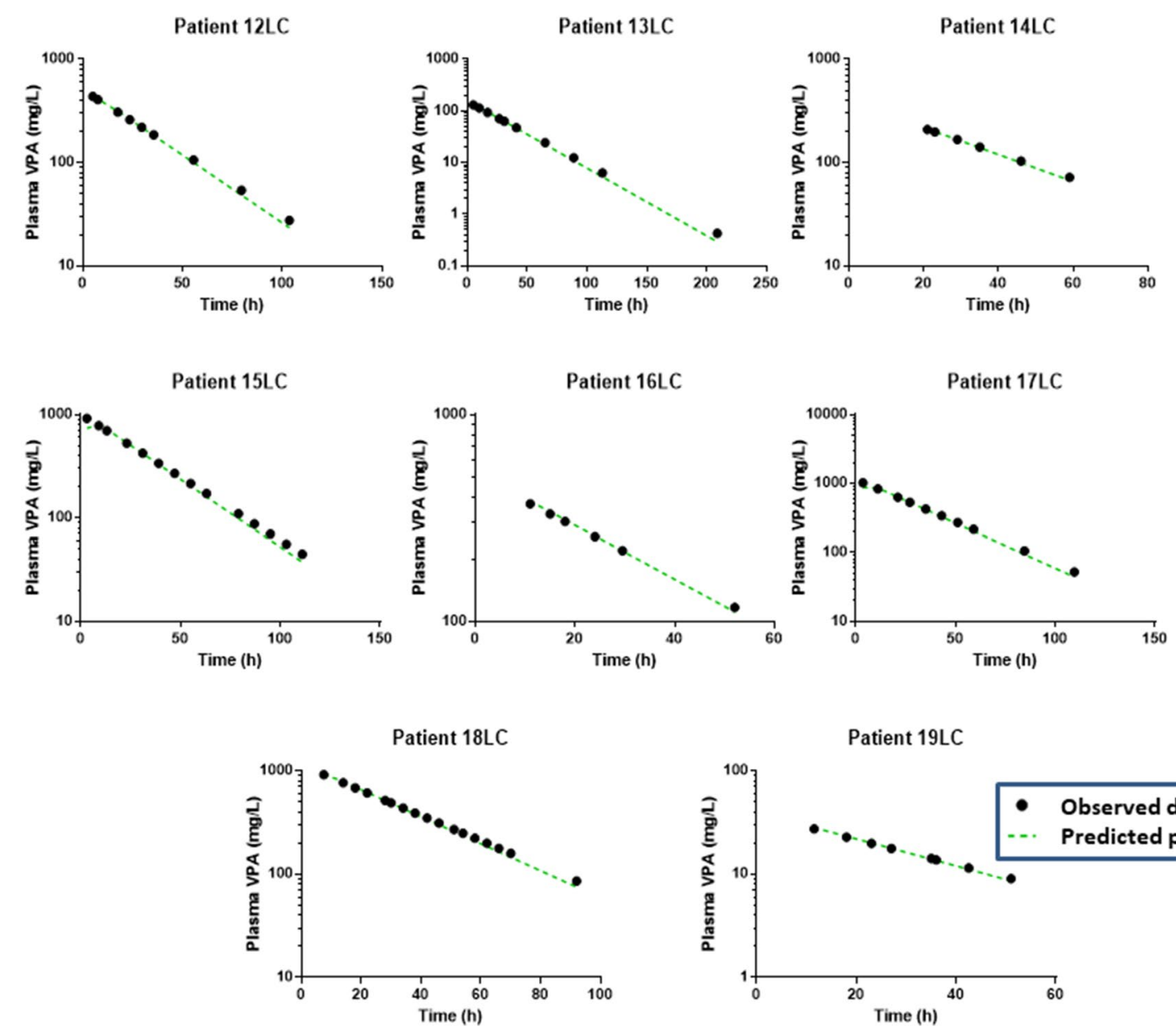

Fig. 2 continued

without available additional descriptive data. Here based on a matched comparative study, we showed that L-carnitine administration was unable to accelerate VPA clearance, speed blood lactate level normalization or limit organ dysfunction. Our findings clearly suggest limiting L-carnitine administration to the most severe VPA-poisoned patients (such as patients with brain edema) and considering alternative therapies that more effectively alter VPA kinetics, such as hemodialysis [8] or meropemen infusion [28].

\section{Study limitations}

Our study presents limitations. Its relatively limited sample size may have underpowered our comparative analyses. The prolonged study duration is a result of VPA poisoning rarity but similarly to the elevated prevalence of co-ingestions, may have introduced confounding factors. Nevertheless, our cases represent real life multidrug poisonings with possible non-identified co-ingested drugs based on the routine screening, risk of drug-drug interactions and no modifications in management with time. However, a case-by-case analysis of the involved toxicants in each patient did not show pertinent drug-drug interactions that could have altered VPA metabolism or elimination. In our approach based on the concentration-effect relationships, we did not account for all possible confounding factors known to influence lactate clearance. In the absence of marked cardiovascular, liver, and renal impairment on ICU admission, we considered that the time-course of blood lactate concentrations could be related almost exclusively to the time-course of VPA concentrations. Although two patients who developed VPA-related brain edema received L-carnitine and improved, no conclusion on its benefits on this major complication could be drawn. Interestingly, the activity and toxicity of the numerous VPA metabolites are poorly understood; none was measured in our study, as assays were not available in our laboratory. Finally, we acknowledge that although confirming all our other findings, the 


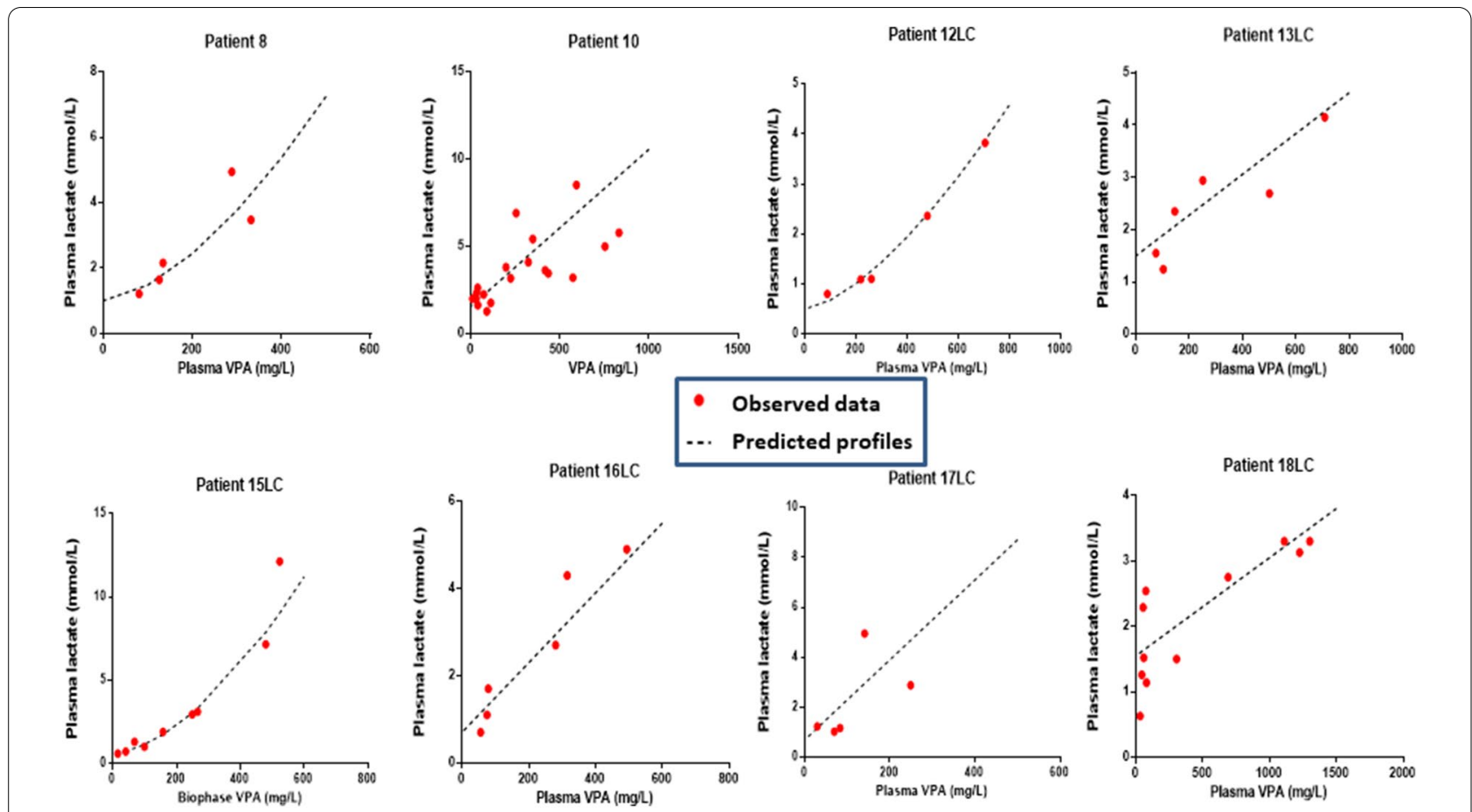

Fig. 3 Individual relationships between blood lactate and plasma valproic acid (VPA) concentrations in eight valproic acid-poisoned patients. LC means treated with L-carnitine
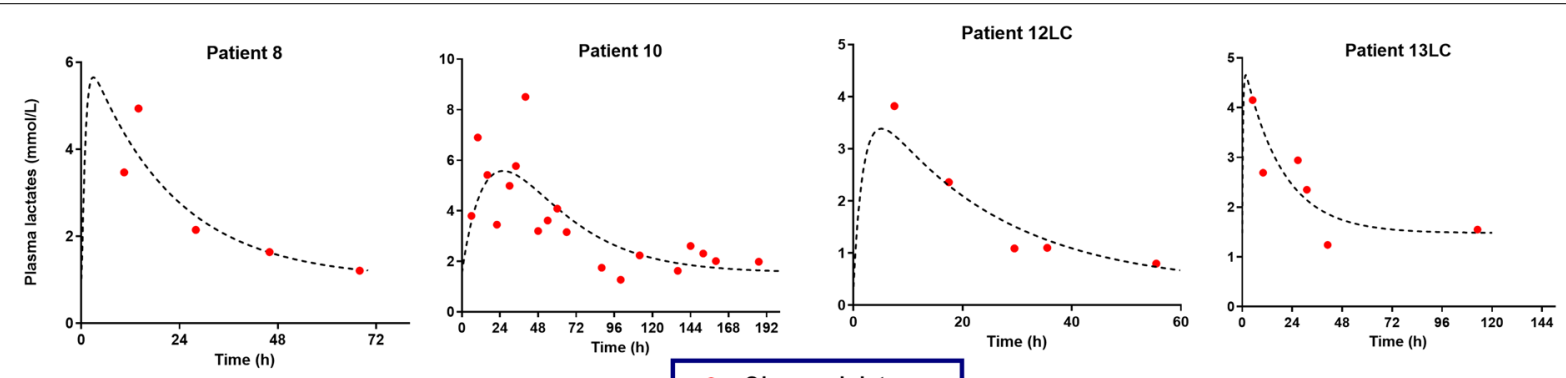

- Observed data

-.. Predicted profiles
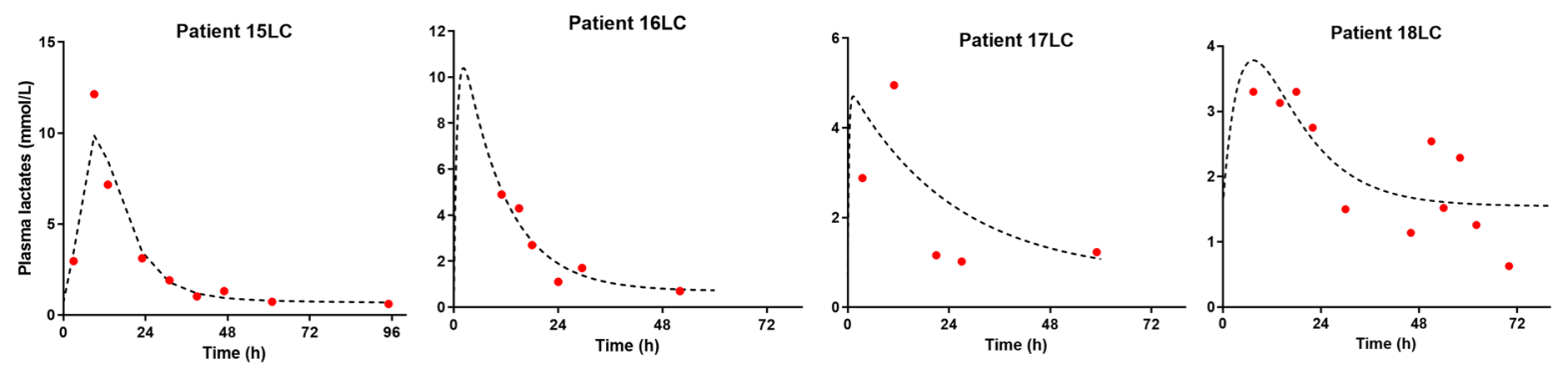

Fig. 4 Individual time-course of blood lactate concentrations in eight valproic acid-poisoned patients. LC means treated with L-carnitine

multivariate analysis of the effectiveness of L-carnitine in VPA poisoning should be interpreted with cautions given the number of variables entered in the model for a small number of outcomes. Likewise, we could not evaluate L-carnitine effects on plasma ammonia, which measurement was not readily available in our institution, thus limiting its repetitive determination. Therefore, due to such limitations, we strongly believe that 
a randomized placebo-controlled multicenter clinical study is warranted to definitively establish the exact role of L-carnitine in VPA poisoning. However, despite no evidence to support its benefits but due to its excellent tolerance and low cost, we believe that L-carnitine administration should still be considered in the meantime on a case-by-case basis, without postponing or substituting supportive care.

\section{Conclusion}

VPA poisoning is responsible for life-threatening manifestations with mildly prolonged VPA elimination. Our findings suggest no benefits of L-carnitine administration on VPA clearance, hyperlactatemia resolution or organ function improvement in VPA-poisoned patients. However, the definitive evaluation of L-carnitine benefits requires a multicenter randomized controlled trial.

\section{Abbreviations}

VPA: Valproic acid; ICU: Intensive care unit; SAPS II: Simplified Acute Physiology Score II; SOFA: Sequential Organ Failure Assessment; $t_{1 / 2}$ : Elimination half-life.

\section{Supplementary Information}

The online version contains supplementary material available at https://doi. org/10.1186/s13613-022-00984-z.

Additional file 1: Table S1. Descriptive analysis of different subgroups of valproic acid-poisoned patients. Table S2. Propensity score used in the multivariate analysis to establish the contribution of L-carnitine to valproic acid-poisoned patient management. Table S3. Multivariate analysis evaluating the effect of L-carnitine administration on the outcome of valproic acid-poisoned patients.

\section{Acknowledgements}

The authors would like to thank Mrs. Alison Good (Scotland, UK) for her helpful review of the manuscript.

\section{Authors' contributions}

BM conceived and designed the study. PN, ASG, IM, ND, SV and BM managed the patients and collected the data. $\mathrm{HG}$ and LL performed the toxicological analysis. LC performed the different modelling. PN and BM performed the analysis and interpretation of data. PN, LC and BM drafted the manuscript. All authors reviewed the manuscript and agreed with the final version and findings. All authors read and approved the final manuscript.

\section{Funding}

This research received no specific grant from any funding agency in the public, commercial, or not-for-profit sectors.

\section{Availability of data and materials}

The data set used during the current study are available from the corresponding author on reasonable request.

\section{Declarations}

\section{Ethics approval and consent to participate}

Approval for this study was obtained from the ethics committee of the French Society of Intensive Care (protocol number, FICS20020231). The study was declared to the Commission Nationale de l'Informatique et des Libertés (declaration number, 2067659). Appropriate information was given to the patients and next of kin. Written informed consent was waived, since no specific intervention related to the research was performed.

\section{Consent for publication}

Not applicable.

\section{Competing interests}

The authors declare that they have no conflicts of interest related to the submitted work.

\section{Author details}

${ }^{1}$ Department of Medical and Toxicological Critical Care, Federation of Toxicology APHP, Lariboisière Hospital, 2 Rue Ambroise Paré, 75010 Paris, France. ${ }^{2}$ University of Paris, Inserm UMRS-1144, Paris, France. ${ }^{3}$ National Egyptian Center for Toxicological Researches, Faculty of Medicine, Cairo University, Cairo, Egypt. ${ }^{4}$ Laboratory of Toxicology, Lariboisière Hospital, Paris, France.

Received: 28 September 2021 Accepted: 14 January 2022

Published online: 29 January 2022

\section{References}

1. Methaneethorn J. A systematic review of population pharmacokinetics of valproic acid. Br J Clin Pharmacol. 2018;84:816-34.

2. Gummin DD, Mowry JB, Beuhler MC, Spyker DA, Brooks DE, Dibert KW, et al. 2019 Annual Report of the American Association of Poison Control Centers' National Poison Data System (NPDS): 37th Annual Report. Clin Toxicol (Phila). 2020;58:1360-541.

3. Sztajnkrycer MD. Valproic acid toxicity: overview and management. J Toxicol Clin Toxicol. 2002:40:789-801.

4. Eyer F, Felgenhauer N, Gempel K, Steimer W, Gerbitz KD, Zilker T. Acute valproate poisoning: pharmacokinetics, alteration in fatty acid metabolism, and changes during therapy. J Clin Psychopharmacol. 2005:25:376-80.

5. Spiller HA, Krenzelok EP, Klein-Schwartz W, Winter ML, Weber JA, Sollee $D R$, et al. Multicenter case series of valproic acid ingestion: serum concentrations and toxicity. J Toxicol Clin Toxicol. 2000;38:755-60.

6. Shadnia S, Amiri H, Hassanian-Moghaddam H, Rezai M, Vasei Z, Ghodrati $\mathrm{N}$, Zamani N. Favorable results after conservative management of 316 valproate intoxicated patients. J Res Med Sci. 2015;20:656-61.

7. Thanacoody RH. Extracorporeal elimination in acute valproic acid poisoning. Clin Toxicol (Phila). 2009;47:609-16.

8. Ghannoum M, Laliberté M, Nolin TD, MacTier R, Lavergne V, Hoffman RS, et al. Extracorporeal treatment for valproic acid poisoning: systematic review and recommendations from the EXTRIP workgroup. Clin Toxicol (Phila). 2015;53:454-65.

9. Mégarbane B, Oberlin M, Alvarez JC, Balen F, Beaune S, Bédry R, et al. Management of pharmaceutical and recreational drug poisoning. Ann Intensive Care. 2020;10:157.

10. Perrott J, Murphy NG, Zed PJ. L-carnitine for acute valproic acid overdose: a systematic review of published cases. Ann Pharmacother. 2010:44:1287-93.

11. Mock CM, Schwetschenau KH. Levocarnitine for valproic-acidinduced hyperammonemic encephalopathy. Am J Health Syst Pharm. 2012;69:35-9.

12. Lheureux PE, Hantson P. Carnitine in the treatment of valproic acidinduced toxicity. Clin Toxicol (Phila). 2009;47:101-11.

13. Le Gall JR, Lemeshow S, Saulnier F. A new simplified acute physiology score (SAPS II) based on a European/North American multicenter study. JAMA. 1993;270:2957-63.

14. Vincent JL, Moreno R, Takala J, Willatts S, De Mendonça A, Bruining $H$, et al. The SOFA (Sepsis-related Organ Failure Assessment) score to describe organ dysfunction/failure. On behalf of the Working Group on Sepsis-Related Problems of the European Society of Intensive Care Medicine. Intensive Care Med. 1996;22:707-10.

15. Khwaja A. KDIGO clinical practice guidelines for acute kidney injury. Nephron Clin Pract. 2012;120:c179-84. 
16. Laub MC, Paetzke-Brunner I, Jaeger G. Serum carnitine during valproic acid therapy. Epilepsia. 1986;27:559-62.

17. Silva MF, Aires CC, Luis PB, Ruiter JP, Ijlst L, Duran M, et al. Valproic acid metabolism and its effects on mitochondrial fatty acid oxidation: a review. J Inherit Metab Dis. 2008;31:205-16.

18. Felker D, Lynn A, Wang S, Johnson DE. Evidence for a potential protective effect of carnitine-pantothenic acid co-treatment on valproic acidinduced hepatotoxicity. Expert Rev Clin Pharmacol. 2014;7:211-8.

19. Wilimowska J, Florek E, Piekoszewski W. Disposition of valproic acid in self-poisoned adults. Basic Clin Pharmacol Toxicol. 2006;99:22-6.

20. Jawień W, Wilimowska J, Kłys M, Piekoszewski W. Population pharmacokinetic modelling of valproic acid and its selected metabolites in acute VPA poisoning. Pharmacol Rep. 2017;69:340-9.

21. Bohles $H$, Sewell AC, Wenzel D. The effect of carnitine supplementation in valproate-induced hyperammonaemia. Acta Paediatr. 1996;85:446-9.

22. Bohan TP, Helton E, McDonald I, Konig S, Gazitt S, Sugimoto T, et al. Effect of L-carnitine treatment for valproate-induced hepatotoxicity. Neurology. 2001;56:1405-9.

23. Glatstein M, Bonifacio Rino P, de Pinho S, Scolnik D, Pivko-Levi D, Hoyte C. Levocarnitine for the treatment of valproic acid-induced hyperammonemic encephalopathy in children: the experience of a large, tertiary care pediatric hospital and a poison center. Am J Ther. 2019;26:e344-9.

24. Chopra A, Kolla BP, Mansukhani MP, Netzel P, Frye MA. Valproate-induced hyperammonemic encephalopathy: an update on risk factors, clinical correlates and management. Gen Hosp Psychiatry. 2012;34:290-8.

25. LoVecchio F, Shriki J, Samaddar R. L-carnitine was safely administered in the setting of valproate toxicity. Am J Emerg Med. 2005;23:321-2.

26. Sakemi K, Hayasaka K, Tahara M, Sanada Y, Takada G. The effect of carnitine on the metabolism of valproic acid in epileptic patients. Tohoku J Exp Med. 1992;167:89-92.

27. Tincu R, Cobilinschi C, Tomescu D, Ghiorghiu Z, Macovei R. Benefits of L-carnitine in valproic acid induced en-cephalopathy [Abstract P331]. Crit Care. 2016;20(Supp2):134.

28. Thomas C, Priano J, Smith TL. Meropenem as an antidote for intentional valproic acid overdose. Am J Emerg Med. 2020;38(3):690.e1-690.e2.

\section{Publisher's Note}

Springer Nature remains neutral with regard to jurisdictional claims in published maps and institutional affiliations.

\section{Submit your manuscript to a SpringerOpen ${ }^{\circ}$ journal and benefit from:}

- Convenient online submission

- Rigorous peer review

- Open access: articles freely available online

- High visibility within the field

- Retaining the copyright to your article 